\title{
Young pre-low-mass X-ray binaries in the propeller phase
}

\section{Nature of the 6.7-h periodic X-ray source 1E 161348-5055 in RCW 103}

\author{
H. Bhadkamkar and P. Ghosh
}

\begin{abstract}
Department of Astronomy \& Astrophysics, Tata Institute of Fundamental Research, Mumbai 400 005, India e-mail: [pranab; harshalhb]@tifr.res.in
\end{abstract}

Received 21 May 2009 / Accepted 23 August 2009

\begin{abstract}
Context. Discovery of the 6.7-h periodicity in the X-ray source 1E 161348-5055 in RCW 103 has led to investigations of the nature of this periodicity.

Aims. To explore a model for 1E 161348-5055 wherein a fast-spinning neutron star with a magnetic field $\sim 10^{12} \mathrm{G}$ in a young prelow-mass X-ray Binary (pre-LMXB) with an eccentric orbit of period $6.7 \mathrm{~h}$ operates in the "propeller" phase.

Methods. The 6.7-h light curve of 1E 161348-5055 is modeled in terms of orbitally-modulated mass transfer through a viscous accretion disk and subsequent propeller emission. Formation of eccentric binaries in supernovae and their subsequent tidal evolution are studied.

Results. The light curve of 1E 161348-5055 can be quantitatively accounted for by models of propeller torques of both IllarionovSunyaev type and Romanova-Lovelace et al. type, and spectral and other properties are also in agreement. Formation and evolution of model systems are shown to be in accordance both with standard theories and with X-ray observations of 1E 161348-5055.

Conclusions. The pre-LMXB model for 1E 161348-5055 and similar sources agrees with observation. Distinguishing features between this model and the recently-proposed magnetar model need to be explored.
\end{abstract}

Key words. X-rays: binaries - stars: neutron - stars: evolution - accretion, accretion disks - ISM: supernova remnants -

X-rays: general

\section{Introduction}

The point soft X-ray source 1E 161348-5055 (henceforth 1E) near the center of the young ( 2000 y old) supernova remnant (SNR) RCW 103 has attracted much attention lately, following the discovery of a strong $6.67 \mathrm{~h}$ periodic modulation in $1 \mathrm{E}$ by de Luca et al. (2006, henceforth dL06) from a deep $X M M-N e w t o n$ observation of the source in 2005 . 1E was discovered in 1980 (Touhy \& Garmire 1980) as a soft Einstein X-ray source. The original interpretation as an isolated neutron star was found to be untenable in view of subsequent discovery by other X-ray satellites (e.g., ROSAT, ASCA, Chandra) of the large variability of $1 \mathrm{E}$ on the timesacle of a few years (dL06). A periodicity at $\sim 6 \mathrm{~h}$ was first hinted at by Chandra observations, but the first clear, strong detection came from the above 2005 observations of dL06, who also showed the existence of this periodicity in the data from earlier 2001 observations of $1 \mathrm{E}$ with $X M M-N e w t o n$, when the source luminosity was higher by a factor $\sim 6$ during the course of its sequence of several-year timescale outbursts nentioned above, documented by these authors from archival data.

The nature of the above $6.67 \mathrm{~h}$ periodicity is an interesting question, on which preliminary discussions were reported in dL06. Recently, Pizzolato et al. (2008, henceforth P08) have proposed a model for the $1 \mathrm{E}$ system wherein it is a close binary consisting of a magnetar, i.e., a neutron star with a superstrong magnetic field $\sim 10^{15} \mathrm{G}$, and a low-mass companion. The $6.67 \mathrm{~h}$ periodicity is identified in this model with the spin period of the neutron star, to which this young neutron star has been spun down in such a short time by the torques associated with its enormous magnetic field. This period has also been proposed by P08 to be in close synchronism with the orbital period of the binary, in analogy with what is believed to be happening in Polar Cataclysmic Variables or AM Her-type systems. The observed $\mathrm{X}$-ray emission from $1 \mathrm{E}$ is that from the magnetar in this model.

In this paper, we explore an alternative model for the $1 \mathrm{E}$ system wherein it is a close binary system consisting of a young neutron star with a canonical magnetic field $\sim 10^{12} \mathrm{G}$, and a lowmass companion, i.e., a pre-low-mass X-ray Binary (henceforth pre-LMXB), such as are believed to be the standard progenitors of Low-mass X-ray Binaries (henceforth LMXBs). Such pre-LMXBs are born after the common-envelope (CE) evolution phase of the original progenitor binary system consisting of a massive star and a low-mass companion, which leads to the formation of a binary consisting of the He-core of the original massive star and the low-mass companion (Ghosh 2007, and references therein). The He-star susequently explodes in a supernova, leading to a neutron star in orbit with a lowmass companion, i.e., the pre-LMXB referred to above. This is the standard He-star supernova scenario for the formation of LMXBs (Ghosh 2007, and references therein). The $6.67 \mathrm{~h}$ periodicity is identified in our model with the orbital period of the binary. In our model the young neutron star is still spinning very rapidly, with a canonical spin period $\sim 10-100 \mathrm{~ms}$, and is operating in the "propeller" regime, wherein any matter approaching the fast-rotating magnetosphere of the neutron star is expelled by the energy and angular momentum deposited into it through its interaction with the magnetospheric boundary (Illarionov \& Sunyaev 1975, henceforth IS75; Davies et al. 1979; Davies \& Pringle 1981; Illarionov \& Kompaneets 1990; 
Mineshige et al. 1991; Illarionov et al. 1993; Ghosh 1995, and references therein, henceforth G95; Lovelace et al. 1999, henceforth LRB99; Romanova et al. 2004, 2005, henceforth RUKL05; Ustyugova et al. 2006, henceforth UKRL06).

The observed X-ray emission from $1 \mathrm{E}$ in our model is that from the propeller: indeed, it is well-known that soft X-ray transients (SXRTs) like Aquila X-1 and others (see Sect. 7.1) go through low/quiescent states during the decay of their outbursts, during which their luminosities and spectral properties are very similar to those of $1 \mathrm{E}$, and the neutron stars in them are believed to be in the propeller regime (Campana et al. 1998; Stella et al. 2000). The observed $6.67 \mathrm{~h}$ periodicity in our model is due to the orbital modulation of the supersonic propeller, which is caused by the orbital modulation of the mass-transfer rate in the eccentric binary orbit of a young system like 1E. It is well-known that young post-SN binaries with low-mass companions like $1 \mathrm{E}$ are almost certain to have eccentric orbits, due to the large eccentricities produced in such systems in the $\mathrm{SN}$ explosion (see Sect. 6.1) and the duration of the subsequent tidal circularization compared to the ages of systems like 1E (see Sect. 6.2). By contrast, SXRTs are believed to be old LMXB systems with circular orbits, where such modulation will not occur.

We show in this work that the $6.67 \mathrm{~h}$ light curve of $1 \mathrm{E}$ can be accounted for quantitatively by our model for propeller torques of both Illarionov-Sunyaev type and RomanovaLovelace et al. type (see Sect. 2), and that the observed spectral and other characteristics are also in general agreement with our overall picture. Thus, further diagnostic features need to be explored in order to distinguish between our model and the magnetar model as a viable description of this and similar sources.

\section{Propeller phase in pre-LMXBs}

In a pre-low-mass X-ray Binary (pre-LMXB: see above), the newborn, fast-rotating neutron star is unable at first to accrete the matter that is being transferred from the companion through the inner Lagrangian point $L_{1}$, because of the fast rotation of the neutron star (IS75, Davies et al. 1979; Davies \& Pringle 1981; Illarionov \& Kompaneets 1990; Mineshige et al. 1991; Illarionov et al. 1993, G95, LRB99; Romanova et al. 2004, RUKL05, UKRL06). Because of its large angular momentum, this matter forms an accretion disk and reaches the magnetospheric boundary of the magnetized neutron star, whereupon this ionized matter interacts with the fast-rotating neutron star's magnetic field, and the energy and angular momentum deposited in it by magnetic stresses associated with this fast-rotating magnetic field expel it. This is the propeller phase of the system (IS75), during which the neutron star spins down as it loses angular momentum and rotational energy. During this propeller phase, the disk matter at the magnetospheric boundary is shockheated as the "vanes" of the supersonic propeller (IS75) hit it, and the hot matter emits in the soft X-ray band. This emission appears unmodulated at the neutron-star spin frequency (as opposed to the X-ray emission from canonical accretion-powered pulsars, which comes from the neutron-star surface) to a distant observer, who sees only the total emission from the heated matter at the magnetospheric boundary. Observations of transient low-mass X-ray binaries (i.e., the soft X-ray transients or SXRTs) like Aquila X-1 (Campana et al. 1998) and SXJ1808.43658 (Stella et al. 2000) in quiescence, when the neutron stars in them are thought to be operating in the propeller phase, amply confirm this point.

The propeller luminosity $L$ during the above phase is given by $L=N \omega$, where $N$ is the propeller torque acting on the neutron star and $\omega$ is its spin angular velocity. The propeller torque $N$ was first estimated by IS75 in their pioneering suggestion of this mechanism, and subsequent work over approximately the next two decades considered variations of this torque under different circumstances, as summarized in G95. These works addressed themselves largely to quasi-spherical accretion, and we shall call this kind of propeller torque the Illarionov-Sunyaev type (or IS-type for short) torque, which was widely used in that time-frame in propeller spindown calculations. In the 2000s, Romanova, Lovelace and co-authors reported a series of calculations of the propeller effect for disk-accreting magnetic stars based on their numerical MHD simulations (Romanova et al. 2004, RUKL05, UKRL06; also see the analytic estimates in LRB99). We shall call the propeller torque obtained from this line of work the Romanova-Lovelace et al. type (or RUKL-type for short) torque. In this work, we shall consider both IS-type and RUKL-type propeller torques for the problem at hand.

Consider IS-type torques first. For such fast-rotating neutron stars as we are concerned with in this work, the propeller operates in the supersonic regime, and its torque is given by (G95 and the references therein),

$N=\frac{1}{6} \frac{\mu^{2} \omega^{2}}{G M_{x}} \frac{\Omega\left(r_{\mathrm{m}}\right)}{\omega}$

In Eq. (1), $\Omega\left(r_{\mathrm{m}}\right)$ is the Keplerian angular velocity at the magnetospheric radius $r_{\mathrm{m}}, \mu$ is the magnetic moment of neutron star and $M_{x}$ is its mass. Combining this equation with the standard expression for the magnetospheric radius (Ghosh 2007), viz.,

$r_{\mathrm{m}}=\left[\frac{\mu^{2}}{\dot{M} \sqrt{2 G M_{x}}}\right]^{\frac{2}{7}}$,

where $\dot{M}$ is the rate at which transferred matter arrives at the magnetospheric boundary, we obtain the following expression for the propeller luminosity:

$L_{35} \approx 5 \dot{M}_{14}^{\frac{3}{7}}\left(P_{\text {spin }} / 0.1 \mathrm{~s}\right)^{-2} \mu_{30}^{\frac{8}{7}} m_{x}^{\frac{-2}{7}}$.

In Eq. (3), $\dot{M}_{14}$ is $\dot{M}$ in units of $10^{14} \mathrm{~g} \mathrm{~s}^{-1}, L_{35}$ is $L$ in the units of $10^{35} \mathrm{erg} \mathrm{s}^{-1}, P_{\text {spin }}$ is the neutron-star spin period, $\mu_{30}$ is the neutron-star magnetic moment in units of $10^{30} \mathrm{G} \mathrm{cm}^{3}$, and $m_{x}$ is the neutron-star mass in units of solar mass. As neutron stars are thought to have $P_{\text {spin }} \sim 0.01-0.1 \mathrm{~s}$ at birth, and as the propeller phase is thought to end when the spin period is longer than $P_{\text {spin }} \sim 0.1-1 \mathrm{~s}$, we have made the canonical choice for the expected scale of $P_{\text {spin }}$ in systems like 1E. Equation (3) clearly shows how the propeller luminosity scales with the mass-arrival rate $\dot{M}$, and essential neutron-star properties, namely, its spin pe$\operatorname{riod} P_{\text {spin }}$, its magnetic moment $\mu$, and its mass $M_{x}$.

Now consider RUKL-type torques. These authors summarized the results of some of their extensive MHD simulations in RUKL05 and UKRL06 in terms of power-law fits to these results, showing that the scaling of the total propeller torque $N$ with the magnetic moment $\mu$ and the spin rate $\omega$ of the neutron star was

$N \propto \mu^{1.1} \omega^{2}$.

However, the scaling of $N$ with $\dot{M}$ was not available from the above references, because only the parameters $\mu$ and $\omega$ (and also the turbulence and magnetic diffusivity parameters of the disk: see below) seem to have been varied in the series of simulations reported in these references. In order to estimate the scaling of $N$ 
with $\dot{M}$ for RUKL-type torques, we proceeded in the following way.

First, we did an analytic estimate in the following manner. In their analytic study, LRB99 argued that the radius $r_{\mathrm{d}}$ of the inner edge of the disk should depend on the stellar rotation rate $\omega$ in addition to the parameters $\mu$ and $\dot{M}$ that $r_{\mathrm{m}}$ (see above) depended upon. The scaling with $\omega, \mu$, and $\dot{M}$ that these authors derived was revised in UKRL06, the final result being given as $r_{\mathrm{d}} \propto \mu^{1 / 2} \dot{M}^{-1 / 4} \omega^{-1 / 4}$. (Note the closeness of the scalings with $\mu$ and $\dot{M}$ with those which apply to $r_{\mathrm{m}}$, as given above.)

In a simple first approach, if we argue that a reasonable estimate of the torque scalings may be obtained by replacing $r_{\mathrm{m}}$ with $r_{\mathrm{d}}$ in Eq. (1) for disk accretion, we arrive at the scaling

$N \propto \mu^{5 / 4} \omega^{11 / 8} \dot{M}^{3 / 8}$

for RUKL-type torques. Noticing the qualitative similarity of the the scalings with $\mu$ and $\omega$ in Eq. (5) with those of the actual RUKL-type torque given in Eq. (4), and furthermore the quantitative closeness for the scaling with $\mu$, we argued that the best estimate would be to use the scalings of Eq. (4) for $\mu$ and $\omega$, and the scaling of Eq. (5) for $\dot{M}$, thus arriving at a suggested scaling for the RUKL-type torque as

$N \propto \mu^{1.1} \omega^{2} \dot{M}^{3 / 8}$.

Before proceeding further, we recognized that RUKL-type torques may arise from more complicated interactions than are describable by the above arguments, and so attempted to verify the above $\dot{M}$ scaling by further comparison with RUKL results. To this end, we noted the correlated variations of $N$ and $\dot{M}$ recorded in Fig. 4 of Romanova et al. (2004), and fitted the two prominent peaks in $N$ and $\dot{M}$ at the extreme right of this figure to a power law. This gave an exponent $\approx 0.37$, coincident with that in Eq. (6) within errors of determination. With this support, we use the scalings of Eq. (6) for the RUKL-type torque in this work, deferring further considerations to future publications.

In order to obtain the dimensional values of the RUKL-type propeller torques and related variables, we now insert the reference units for the RUKL simulations given in RUKL05 and UKRL06, thus obtaining for the torque:

$N_{33} \approx 0.87 \mu_{30}^{1.1}\left(P_{\text {spin }} / 0.01 \mathrm{~s}\right)^{-2} \dot{M}_{14}^{3 / 8}$.

Here, $N_{33}$ is the propeller torque in units of $10^{33} \mathrm{~g} \mathrm{~cm}^{2} \mathrm{~s}^{-2}$, the units of other variables are as before, and we have kept the values of the turbulence and magnetic diffusivity parameters of the accretion disk in RUKL-type models at the canonical values given in RUKL05 and UKRL06. The RUKL propeller luminosity $L$ is then obtained in a straightforward manner as

$L_{35} \approx 5.5 \dot{M}_{14}^{\frac{3}{8}}\left(P_{\text {spin }} / 0.01 \mathrm{~s}\right)^{-3} \mu_{30}^{1.1}$.

In Eq. (8), the units of all variables are as before.

Comparison of Eqs. (3) and (8) immediately leads to the following conclusions about IS-type and RUKL-type propeller luminosities. First, the scalings with $\mu$ and $\dot{M}$ are almost identical for the two types. Secondly, the scaling with the neutron-star spin period $P_{\text {spin }}$ is stronger $(-3$ instead of -2$)$ for the RUKLtype than for the IS-type. Finally, for identical values of the variables $\mu, \dot{M}$, and $P_{\text {spin }}$, the RUKL-type propeller luminosity is about three orders of magnitude lower than the IS-type propeller luminosity. Conversely, at fixed values of $\mu$ and $\dot{M}$, roughly equal luminosities are given by the two types if the spin-rate for the RUKL type is about an order of magnitude higher than that for the IS type.
As indicated earlier, in this work we are exploring the properties of such propellers as described above during the relatively early stages of post-supernova binaries containing pre-LMXBs, when the binary orbits are expected to be appreciably eccentric, as explained in Sect. 6.1. In such a system, the mass-transfer rate $\dot{M}_{\text {tr }}$ through the inner Lagrangian point $L_{1}$ is expected to vary periodically with the orbital phase, as detailed below in Sect. 3. This flow of matter forms an accretion disk because of its large specific angular momentum, as explained above, and slow viscous effects in the disk modify the profile of the above periodic modulation (making it less sharp), and the resultant periodic profile is that which is shown by the mass-arrival rate $\dot{M}$ at the neutron star. The propeller luminosity then follows suite, showing a periodic modulation, as described by Eq. (3) for the IS-type torque or Eq. (8) for the RUKL-type torque. In this scenario, therefore, we identify the $6.67 \mathrm{~h}$ period of $1 \mathrm{E}$ with the binary period of a young, eccentric pre-LMXB, which is expected to turn much later into a standard LMXB after passing through further intermediate phases (see Sect. 6.4). In the next section, we give details of the expected nature of the mass-transfer modulation $\dot{M}_{\text {tr }}(\theta)$ at the orbital period.

\section{Orbital modulation of mass transfer}

The problem of orbital modulation of mass transfer in eccentric orbits has been studied by a number of authors over almost three decades now, adopting various approaches appropriate for various aspects of the problem they have studied. These aspects have covered a considerable range, from a scrutiny of the concept of the Roche lobe in an eccentric orbit (Avni 1976), to a study of test-particle motion through numerical integration of the restricted three-body problem at or near periastron passage (Lubow \& Shu 1975), to explicit calculations of orbital phasedependent flow through $L_{1}$ from a suitably-modeled stellar envelope (Joss \& Rappaport 1984, and references therein). For our purposes here, we have adopted the results of the calculations described by Brown \& Boyle (1984, hereafter BB): these authors described the flow through $L_{1}$ from the atmosphere of the lobe-filling companion with a scale height $H$ as a sort of nozzle flow through the inner Lagrangian point, integrating over a Maxwellian distribution of velocities (characterized by thermal velocity scale $v_{\mathrm{T}}$ ) for the stellar matter. Their final result for the rate of mass transfer as a function of the true anomaly $\theta$ is given by:

$\dot{M}_{\mathrm{tr}}(\theta)=\dot{M}_{0} \frac{\gamma}{\gamma_{\mathrm{p}}} \frac{1+e}{1+e \cos \theta} \exp \left[-\gamma \beta e\left(\frac{1-\cos \theta}{1+e \cos \theta}\right)\right]$.

In Eq. (9), $e$ is the orbital eccentricity, and the dimensionless function $\gamma(\theta)$ is the ratio of the phase-dependent equivalent Roche-lobe radius $R(\theta)$ of the companion to the phase-dependent orbital distance $d(\theta)$ in the eccentric orbit, $\gamma_{\mathrm{p}}$ being the value of $\gamma$ at periastron $(\theta=0)$. From standard geometry of ellipses, $d(\theta)$ is given by:

$d(\theta)=p \frac{1+e}{1+e \cos \theta}$

where $p \equiv a(1-e)$ is periastron distance. Finally, $\beta \equiv p / H$ is the the dimensionless scale-height parameter introduced by BB.

It is convenient to work in terms of the ratio $\gamma$ as it varies relatively slowly with orbital phase (and is, in fact, independent of this phase for a non-rotating companion: see below). The other properties of the binary system that $\gamma$ depends on are (a) the mass 
ratio $Q \equiv M_{\mathrm{c}} / M_{x}, M_{\mathrm{c}}$ being the mass of the low-mass companion; and (b) the rate of rotation $\Omega_{\mathrm{c}}$ of the companion, usually expressed in units of the orbital angular velocity $\Omega_{\mathrm{p}}$ at periastron as $\lambda \equiv \Omega_{\mathrm{c}} / \Omega_{\mathrm{p}}$. The scale $\dot{M}_{0}=\sqrt{2 \pi} \gamma_{\mathrm{p}} p H v_{\mathrm{T}} \rho_{0}$ of the mass-transfer rate in Eq. (9) is set by the above velocity scale $v_{\mathrm{T}}$, the scale-size $p H$ for the effective cross-section of the above "nozzle", and the basic density scale $\rho_{0}$ in the stellar atmosphere.

Prescriptions for $\gamma$ have been given in the 1970s and '80s; we use here the generalized Joss-Rappaport (Joss \& Rappaport 1984) expressions adopted by BB, namely,

$\gamma=A-B \log Q+C(\log Q)^{2}$,

where the coefficients in $\gamma$ are given by:

$$
\left.\begin{array}{l}
A=0.398-0.026 K+0.004 K^{3 / 2} \\
B=-0.264+0.052 K-0.015 K^{3 / 2} \\
C=-0.023-0.005 K
\end{array}\right\}
$$

and the variable $K$ depends on the above rotation parameter $\lambda$ and the orbital phase as:

$$
K=\lambda^{2} \frac{(1+e)^{4}}{(1+e \cos \theta)^{3}} .
$$

From Eqs. (11)-(13), it is clear that, for a non-rotating companion with $K=0, \gamma$ is independent of the orbital phase, and depends only on the mass ratio $Q$. Thus, for a given $Q, R(\theta)$ simply scales with $d(\theta)$ as the eccentric orbit is traversed. It is stellar rotation which modifies the Roche potential in such a way that this simple scaling is broken, and $\gamma$ depends on orbital phase. The phase-dependent factor in $K$ goes back to the original work of Avni (1976). In our present work, we study the limits of (a) no stellar rotation, $\lambda=0$, and (b) synchronous stellar rotation, $\lambda=1$, to cover a range of possibilities (see below). The estimated accuracy in the above prescription for determining equivalent Roche-lobe radii is $\sim 2 \%$.

Detailed models with the mass transfer profile $\dot{M}_{\mathrm{tr}}(\theta)$ given by Eq. (9) are described below. From general considerations, it is clear that this profile peaks at the periastron and that the sharpness of the peak depends on the quantity $\gamma \beta e$. Since $\gamma \sim 1$, and typical values of $\beta$ for the current problem are in the range $10^{2}-10^{3}$ (BB), we see that the profile is expected to be sharply peaked at the periastron even for realtively low values of eccentricity, such as $e \sim 0.2$.

\section{Viscous flow in accretion disks}

Matter transferred through $L_{1}$ into the Roche lobe of the neutron star first forms a ring around the neutron star, the radius $r_{\text {ring }}$ of this ring being related to the specific angular momentum $\ell_{\mathrm{tr}}$ of the transferred matter as (Pringle 1981):

$r_{\text {ring }}=\ell_{\text {tr }}^{2} /\left(G M_{x}\right)$.

Through effective viscous stresses, this ring spreads into an accretion disk, wherein matter slowly spirals inward towards the neutron star as the viscous stresses remove angular momentum from it. The accretion disk extends from its outermost radius $r_{\text {out }}$ inward upto the magnetospheric boundary $r_{\mathrm{m}}$, where the propeller torques expel the matter by depositing energy and angular momentum in it, as explained above.

The rate $\dot{M}$ at which the matter drifting radially inward through the accretion disk arrives at $r_{\mathrm{m}}$ depends, therefore, both on the profile of mass supply $\dot{M}_{\text {tr }}$ at $L_{1}$, as described above, and on the rate of viscous radial drift through the accretion disk, which occurs on a timescale $t_{\text {visc }}$.

In a quasi-steady state, the relation between the two profiles $\dot{M}(t)$ and $\dot{M}_{\mathrm{tr}}(t)$ is of the form

$\dot{M}(t)=\int_{t-N * P_{\mathrm{orb}}}^{t} \dot{M}_{\mathrm{tr}}\left(t_{0}\right) f(\tau) \mathrm{d} t_{0}, \quad$ where $\quad \tau \equiv \frac{t-t_{0}}{t_{\mathrm{visc}}}$

The convolution integral in Eq. (15) describes the viscous drift with the timescale $t_{\mathrm{visc}}$ of the mass supplied to the disk at earlier times $t_{0}$ at the rate $\dot{M}_{\text {tr }}\left(t_{0}\right)$, as indicated above. In principle, the integral extends over all previous times, but in practice it is sufficient to keep track of only about $N$ orbital periods in the past (as the lower limit of integration indicates). This is so because of the rapid fall of of the viscous-evolution profile $f(\tau)$ of the accretion disk at large values of $\tau$ (see below).

Viscous-evolution profiles have been calculated analytically and numerically at various levels of approximation by several authors (Lynden-Bell \& Pringle 1974; Lightman 1974). For our purposes here, we have adopted an analytic approximation of the generic form

$f(\tau)=\tau^{-n} \exp \left(\frac{-n}{\tau}\right)$

introduced and utilized by Pravdo \& Ghosh (2001, hereafter PG). This reference has discussions of earlier analytical and numerical investigations. The generic PG profile in Eq. (16) reaches its maximum at $\tau=1$, and decays subsequently as $\tau^{-n}$. Clearly, therefore, most of the contribution to the above convolution integral comes from those orbital cycles which are closest to the earlier time $t_{0}=t-t_{\text {visc }}$, and $N$ is determined by the sharpness of the fall of the profile, i.e., $n$. In our computations, we estimated the optimal values of $N$ by running test cases with increasing values of $N$ until the desired accuracy was obtained. For example, in the best-fit case reported below, we found that $N=9$ gave an accuracy of $\approx 10 \%$, while $N=15$ gave an accuracy of $\approx 1 \%$. Given the error bars on the data points in the observed light curve, further accuracy was unnecessary.

The following generic feature of viscous evolution of accretion disks is a key aspect of the phenomenon we are exploring here. Whereas the orbital modulation of the mass-supply rate $\dot{M}_{\text {tr }}(t)$ to the disk at its outer radius $r_{\text {out }}$ is expected to sharply peaked at periastron for typical values of the scale height in the companion's atmosphere, as above, the viscous drift of matter through the accretion disk would decrease the sharpness of this modulation, since variations on timescales much shorter than $t_{\text {visc }}$ tend to be "washed out" by viscous diffusion. This is what makes the orbital modulation of the mass-arrival rate $\dot{M}(t)$ at the disk's inner radius $r_{\mathrm{m}}$ gentler, and therefore also the modulation of the propeller luminosity $L(t)$, leading naturally to light curves of the form observed in 1E. Quantitative details follow.

\section{Model light curves}

We constructed model light curves for $1 \mathrm{E}$ by combining the model of mass transfer described in Sect. 3 with that of viscous flow through the accretion disk described in Sect. 4. We then fitted these models to the observed light curve of $1 \mathrm{E}$ in 2005 (dL06). The fitting parameters were $(\beta, e)$, which come from the above BB mass-transfer model in elliptic orbit, and also $\left(t_{\mathrm{visc}}\right.$, $n$ ), which come from the above PG parametrized description of viscous evolution of accretion disks. In this introductory work, we kept $\beta$ constant at a canonical value of $\beta=10^{3}$ (BB), and varied the parameters $e, t_{\text {visc }}$, and $n$ to obtain acceptable fits. For 

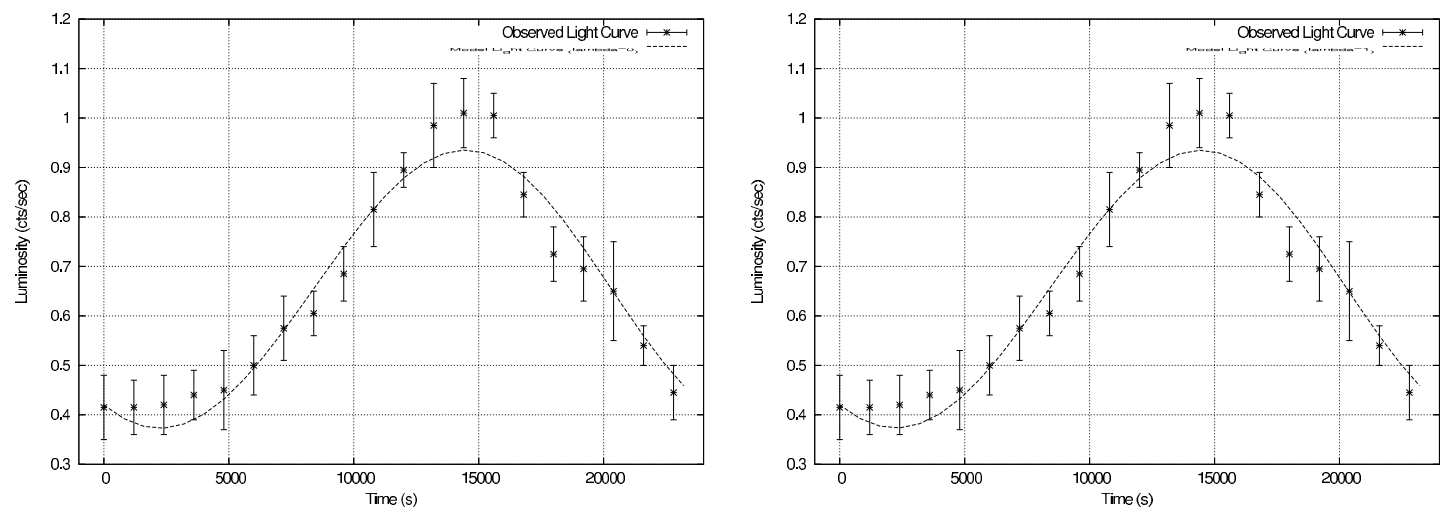

Fig. 1. X-ray light curve of 1E. Shown is the observed light curve from dL06, superposed on the (common) best-fit model light curve for IS-type and RUKL-type propellers. Left panel: model curve for $\lambda=0$ (nonrotating companion). Right panel: same for $\lambda=1$ (synchronously rotating companion).

Table 1. Best fit model parameters: IS-type torque.

\begin{tabular}{ccc}
\hline \hline Parameter & Best fit value $(\lambda=0)$ & Best fit value $(\lambda=1)$ \\
\hline$\kappa$ & 2.6 & 2.6 \\
viscous-profile index $n$ & 5.04 & 5.04 \\
eccentricity & 0.405 & 0.406 \\
\hline$\chi^{2}$ & 1.013 & 1.012 \\
\hline
\end{tabular}

Table 2. Best fit model parameters: RUKL-type torque.

\begin{tabular}{ccc}
\hline \hline Parameter & Best fit value $(\lambda=0)$ & Best fit value $(\lambda=1)$ \\
\hline$\kappa$ & 2.6 & 2.6 \\
viscous-profile index $n$ & 5.02 & 5.02 \\
eccentricity & 0.400 & 0.401 \\
\hline$\chi^{2}$ & 1.003 & 1.006 \\
\hline
\end{tabular}

the viscous timescale, we found it more convenient to work in terms of the ratio $\kappa \equiv t_{\mathrm{visc}} / P_{\mathrm{orb}}$ of this timescale to the known period $P_{\text {orb }} \approx 6.67 \mathrm{hr}$ of the system, which we of course identify with the orbital period in this model. The ratio $\kappa$ is of immediate physical significance, since it measures the relative importance of viscous diffusion to orbital modulation in the system. For $\kappa \ll 1$, viscous diffusion would be so rapid as to enable the disk flow to adjust to the orbital modulation of mass-supply rate, and flow-rate would essentially follow the supply rate. For $\kappa \gg 1$, on the other hand, the viscous diffusion would be so slow as to wash out any rapid variations in the mass-supply rate, and the modulation would be essentially determined by the disk viscosity. As we see below, $\kappa$ values of a few seem to describe the $1 \mathrm{E}$ system, indicating comparable importance of the two effects in this system.

We fitted model light curves corresponding to both IS-type and RUKL-type torques to the data on $1 \mathrm{E}$, the best-fit values of the parameters being given in Tables 1 and 2. In each case, we have considered both a non-rotating secondary $(\lambda=0)$ and a synchronously-rotating secondary $(\lambda=1)$, as indicated. Note that the best-fit values for the two types of torques are very close to each other, as may have been expected. This is so because the closeness of the scaling of $L$ with $\dot{M}$ between the two types, as discussed in Sect. 2, since only this aspect of the torque is relevant for fitting the profile of the light curve. Other aspects, e.g., the fact that the RUKL-type propeller luminosity is about three orders magnitude below the IS-type propeller lumnosity for identical vaues of $\mu, \dot{M}$ and $\omega$, have important consequences elsewhere, as detailed in Sect. 6.3, but not in this matter. Further, the absolute values of the observed luminosities in the light curves are easily accounted for, e.g., by having the stellar spin rate higher for RUKL-type torques by about a factor of 10 than that for IS-type torques, and $\mu, \dot{M}$ identical for the two types, as the scalings in Eqs. (8) and (3) show. This implies neutron-star spin periods in the range $P_{\text {spin }} \sim 0.01-0.1$ s, i.e., the canonical range for propellers, for both type of torques, as explained in Sect. 1.

This closeness of best-fit parameters is reflected in the bestfit light curves, which are visually essentially identical for the two types of torques. In Fig. 1, we display this common best-fit light curve, superposed on the data on $1 \mathrm{E}$.

Our inferred best-fit value of $\kappa$ in the above tables indicates that the dominant contribution to the convolution integral described in the last section comes from the second and third orbits preceding the time of observation. The corresponding viscous timescale $t_{\text {visc }} \approx 17.3 \mathrm{~h}$ is consistent with a rather thick disk with $h / r \sim 0.1-0.5$ and a canonical value $\sim 0.1-1$ for the disk viscosity parameter (Shakura \& Sunyaev 1973). This seems consistent with the results of the RUKL numerical simulations. Note also that the best-fit value of the viscous-profile index $n$ is consistent with the range of values $n \sim 4-5$ generally expected for neutronstar systems, as per the discussion given in PG. Indeed, we found that values of $n$ in the above range generally worked for the $1 \mathrm{E}$ system. Regarding the orbital eccentricity $e$, the best-fit values are as given in the tables, and we found that values of the eccentricity $e$ in the range $\sim 0.35-0.45$ genearlly worked for the $1 \mathrm{E}$ system: we discuss this in the next section. It is clear, therefore, that the model explored in this paper can account quantitatively for the observed 1E light curve in 2005, for both IS-type and RUKL-type torques. We discuss in Sect. 7.2 possible reasons for the apparently different, "jagged" light curve hinted at by the 2001 observations of this system (dL06).

\section{Formation and evolution of prototype systems}

As indicated in Sect. 1, we are exploring in this work a model for systems like $1 \mathrm{E}$ wherein the binary system of a He-star and a low-mass star (left after completion of the CE evolution phase in which the extensive envelope of the evolved primary has been expelled and its He-core left behind) produces the pre-LMXB when the He-star explodes in a supernova ( $\mathrm{SN}$ ), leading to a newborn neutron star with a low-mass companion. Essential features of the formation and subsequent evolution of such systems are, therefore, essential components of this model. We now 
discuss these features in brief, considering in this section first the immediate post-SN status of the system, and then the evolution of this system with the low-mass companion in an eccentric orbit at or near the point of Roche-lobe contact at periastron, producing a system like $1 \mathrm{E}$ where orbitally-modulated mass transfer proceeds through the inner Lagrangian point, and the newborn, fast-spinning neutron star is operating in the propeller regime, expelling this matter instead of accreting. Subsequently, we summarize further evolution of such systems.

\subsection{Immediate post-SN systems}

A major question that concerns us here is the expected eccentricity of systems formed by the $\mathrm{SN}$ in the above scenario, since this eccentricity is crucial for the proposed mechanism. Qualitatively, it is obvious that the immediate post-SN system is almost guaranteed to be highly eccentric, as the mass loss from a typical pre-SN system of, say, a $3 M_{\odot} \mathrm{He}$-star and a $M_{\mathrm{c}}=0.4 M_{\odot}$ low-mass companion (see below) in forming the post-SN system of $M_{x}=1.4 M_{\odot}$ neutron star with its $M_{\mathrm{c}}=0.4 M_{\odot}$ low-mass companion is $1.6 M_{\odot}$, which is close enough to maximum allowed value of mass loss (=half of the initial total mass of $3.4 M_{\odot}$ for zero kick velocity) to ensure that the post-SN orbit would be very eccentric. We shall use these values for the stellar masses throughout the rest of this paper.

To see this quantitatively, we can adapt the extensive calculations of Kalogera, who computed the probability of the formation of X-ray binaries as a funtion of orbital parameters (Kalogera 1996). In the following, we shall use the same masses for the pre- and post-SN system as given above for illustrative purposes. The probability density from Kalogera's work is:

$$
\begin{aligned}
G(\alpha, e)= & \left(\frac{\zeta}{2 \pi \xi^{2}}\right)^{3 / 2} \frac{2 \pi e}{\left[\alpha\left(1-e^{2}\right)\right]^{1 / 2}} \\
& \times\left[\left(\alpha-\frac{1}{1+e}\right)\left(\frac{1}{1-e}-\alpha\right)\right]^{-1 / 2} \\
& \times \exp \left[-\frac{1}{2 \xi^{2}}\left(\zeta \frac{2 \alpha-1}{\alpha}+1\right)\right] I_{\mathrm{o}}(z) .
\end{aligned}
$$

Here,

$z \equiv \frac{\left(\zeta \alpha\left(1-e^{2}\right)\right)^{1 / 2}}{\xi^{2}}$,

and $I_{\mathrm{O}}$ is the modified Bessel function of zeroth order. Further, $\alpha$ is the ratio of semimajor axes of the pre- and post-SN orbits, $\zeta$ is the ratio of the total mass of the post-SN binary to that of the pre-SN one, and $\xi \equiv \sigma / V_{\mathrm{r}}, \sigma$ being the velocity dispersion in the SN kick-velocity, and $V_{\mathrm{r}}$ the orbital velocity of the exploding star relative to its low-mass companion just before the $\mathrm{SN}$ (Kalogera 1996).

In the problem we are studying here, the semimajor axis of the post-SN binary is determined by Kepler's third law from our assumed stellar masses above, and the known orbital period of 1E. However, when there is a kick associated with the SN, the inferred semimajor axis of the pre-SN binary is not determined uniquely by the semimajor axis and the eccentricity of the postSN binary: rather, there is a range of values corresponding to the range of the kick-velocity. Thus, there is a range in the values of $\alpha$ : it is well-known that the allowed range for $\alpha$ is limited from $1 /(1+e)$ to $1 /(1-e)$, these limits being first identified by Flannery and van den Heuvel (1975). Thus, for our purposes, it is aprropriate to integrate $G(\alpha, e)$ over the above allowed range of $\alpha$, and

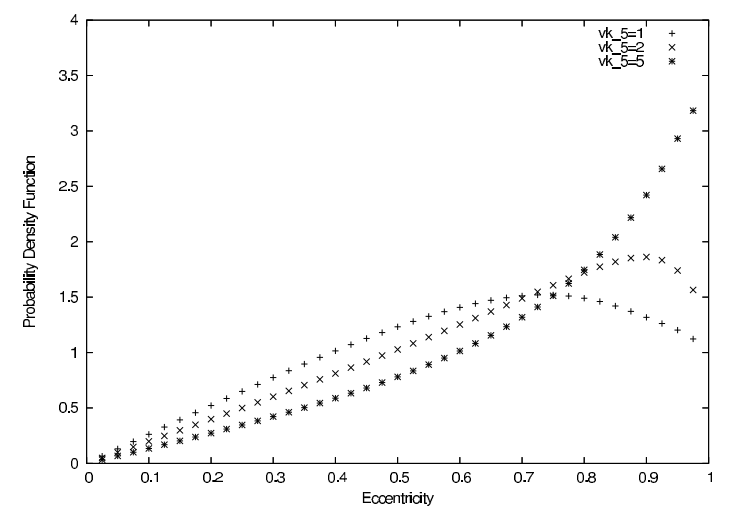

Fig. 2. Formation probability-density $\mathcal{G}(e)$ of immediate post-SN binaries as a function of eccentricity $e$ for various values of the dispersion $\sigma$ in the SN kick velocity (see text). Curves labeled by the value of $v k_{5}=\sigma$ in units of $100 \mathrm{~km} \mathrm{~s}^{-1}$. Each curve so normalized that $\int \mathcal{G}(e) \mathrm{d} e=1$.

display the resultant probability density $\mathcal{G}(e) \equiv \int G(\alpha, e) \mathrm{d} \alpha$ as a function of the eccentricity $e$. We show this in Fig. 2 for various typical values of $\sigma$ as indicated. In this figure, we have used the symbol $v k_{5}$ there to denote $\sigma$ in units of $10^{5} \mathrm{~m} \mathrm{~s}^{-1}=100 \mathrm{~km} \mathrm{~s}^{-1}$, the typical scale for the SN kick dispersion, and we have normalized the probability density $\mathcal{G}(e)$ so that $\int \mathcal{G}(e) \mathrm{d} e=1$ in each case. As explained above, the closeness of the value of $\zeta \approx 0.53$ in this typical case to its lower limit for no binary destruction in the SN (this limit is 0.5 for zero kick velocity) ensures that the probability peaks at a high value of $e$, as Fig. 2 shows. It is clear, therefore, that such a pre-LMXB would generically have a considerable eccentricity at the time of its formation in the SN.

\subsection{Tidal-evolution phase of pre-LMXBs}

The above newly-formed pre-LMXB undergoes tidal evolution, wherein three simultaneous processes occur, namely, (1) tidal circularization, i.e., decrease in the orbital eccentricity $e$; (2) tidal orbit-shrinkage or hardening, i.e., decrease in the orbital semimajor axis $a$; and (3) tidal synchronization, whereby the rotation frequency $\Omega_{\mathrm{c}}$ of the low-mass companion approaches the orbital angular frequency $\Omega \equiv 2 \pi / P_{\text {orb}}$. These processes happen through tidal torques, and their quantitative descriptions pioneered by Zahn $(1977,1978)$ are widely used for calculations: we use them here, as have P08. Complete equations are given in Zahn (1977), and an Erratum was published by Zahn (1978). We have found a further algebraic or transcription error in the original paper, which we describe below, and which seems to have gone unnoticed so far.

Complete formulations for the rates of change of $e, a$, and $\Omega_{\mathrm{c}}$ are given in Zahn (1977), but for our work here we shall utilize a widely-used simplification which comes naturally out of these formulations, namely, that the timescale for tidal synchronization comes out to be much shorter than that for tidal circularization and tidal hardening (see, e.g., Meibom \& Mathieu 2005; P08). This is appropriate, since we shall be interested in this work only in phenomena which occur on the timescales of tidal circularizatuion or longer. Under such circumstances, we can look upon the system as being roughly synchronous at all times, and describe tidal circularization and tidal hardening respectively by Zahn's (1977) Eq. (4.7) and the appropriately 
simplified (i.e., synchronized) version of Zahn's Eq. (4.3), thereby obtaining:

$$
-\frac{1}{e} \frac{\mathrm{d} e}{\mathrm{~d} t}=\frac{63}{4} \frac{k_{2}}{t_{F}} q(1+q)\left(\frac{R}{a}\right)^{8}
$$

and

$$
-\frac{1}{a} \frac{\mathrm{d} a}{\mathrm{~d} t}=114 \frac{k_{2}}{t_{\mathrm{F}}} q(1+q)\left(\frac{R}{a}\right)^{8} e^{2}
$$

In Eqs. (18) and (19), $q \equiv 1 / Q$ in terms of the mass ratio $Q \equiv M_{\mathrm{c}} / M_{x}$ defined above in Sect. 3, $k_{2}$ is the apsidal motion constant for the low-mass companion, and $t_{\mathrm{F}}$ is the "friction time" of Zahn (1977), which, for stars with convective envelopes (as in the present case) is given by Zahn's (1977) pioneering prescription of the turbulent eddy-viscosity timescale $t_{\mathrm{EV}}$ :

$t_{\mathrm{F}} \sim t_{\mathrm{EV}}=\left(M_{\mathrm{c}} R_{\mathrm{c}}^{2} / L_{\mathrm{c}}\right)^{1 / 3}$.

Equations (18) and (19) describe simultaneous tidal circularization and hardening of close binaries, but before presenting our results, we need to correct two errors related to them. First, if we define a circularization timescale $t_{\text {circ }} \equiv-e /(\mathrm{d} e / \mathrm{d} t)$ in the usual way, we get from Eq. (18) the result:

$t_{\text {circ }}=\frac{4}{63} \frac{1}{k_{2} q(1+q)}\left(\frac{M_{\mathrm{c}} R_{\mathrm{c}}^{2}}{L_{\mathrm{c}}}\right)^{1 / 3}\left(\frac{a}{R}\right)^{8}$,

which would be identical to Zahn's (1977) Eq. (4.13), except that the factor of 4 on the right-hand side is missing in Zahn (1977). Unfortunately, this error has propagated over the years into numerous papers, e.g., in P08, in their Eq. $(2)^{1}$ We have corrected this now. Secondly, in an erratum published in 1978, Zahn corrected a few other (generally smaller) numerical errors, of which the one relevant to our work is that the numerical coefficient on the right-hand side of our Eq. (18) should be 21 instead of 63/4. In all calculations reported here, we have made these corrections.

We have integrated Eqs. (18) and (19) numerically for close binary systems like $1 \mathrm{E}$, with values of initial post-SN semimajor axes and eccentricities, $a_{i}$ and $e_{i}$, chosen over a range of plausible values for such systems. We find that, in all cases, the systems circularize and harden in a way that, in the ( $e$ vs. $a$ ) plane, the circularization point is approached in a "cut off" like manner. This is shown in Fig. 3 for a possible prototype 1E-like system, so chosen that the parameters of it evolve to those roughly corresponding to $1 \mathrm{E}$ in $\sim 2000$ years. This cut-off approach is similar to what Meibom \& Mathieu (2005) found. Of course, our detailed shape is slightly different from that of these authors, since they fitted their results to an assumed parameterized distribution shape applicable to observations on a collection of "normal" binaries. These details will be given in a separate publication. For our purposes here, we note that the total time $\tau_{\text {circ }}$ taken to reach this circularization point (Meibom \& Mathieu 2005) can be expressed roughly as:

$\tau_{\text {circ }} \approx \tau_{0}\left(\frac{a_{i}}{R_{\mathrm{c}}}\right)^{8} e_{i}^{-2.55}$

where $a_{i}$ and $e_{i}$ are the initial semimajor axis and eccentricity of the immediate post-SN orbit, and the scale parameter $\tau_{0}$ is given by:

$\tau_{0} \approx \frac{1}{2 k_{2} q(1+q)}\left(\frac{M_{\mathrm{c}} R_{\mathrm{c}}^{2}}{L_{\mathrm{c}}}\right)^{1 / 3}$.

${ }^{1}$ Because of this, the parameters adopted for $1 \mathrm{E}$ by P08 and by ourselves in this work actually give $t_{\text {circ }} \sim 10^{4} \mathrm{yr}$. In our work, we have used the values of the apsidal-motion constant $k_{2}$ given by Landin et al. (2009).

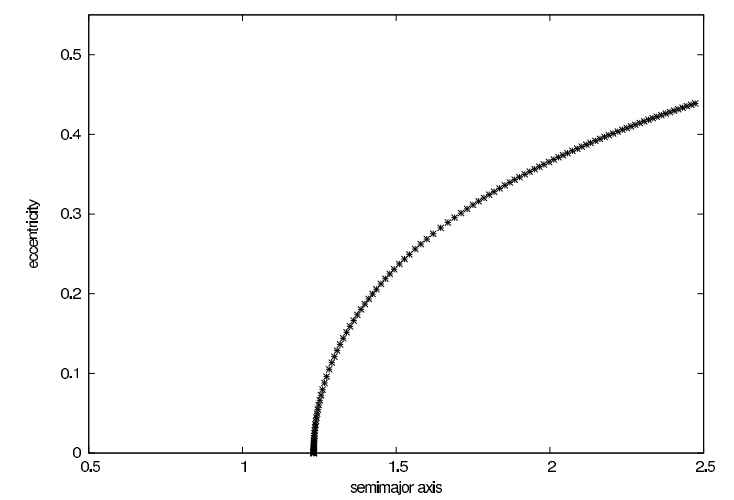

Fig. 3. Tidal evolution of a prototype 1E-like system in the $e$ vs. $a$ plane. Semimajor axis $a$ in units of solar radius. Note the "cut off" like approach to the circularization point (see text).

Equation (22) is a rough analytic fit to the mumerical results, adequate for our purposes. Note that the scale parameter $\tau_{0}$ depends on the companion mass $M_{\mathrm{c}}$, its value being $\tau_{0} \approx 1 \mathrm{yr}$ for the inferred companion mass of $1 \mathrm{E}$.

It is clear from Eq. (22) that circularization is faster for orbits which are born more compact and more eccentric. The scaling with $a$ is straightforward from the above equations of tidal evolution; the scaling with $e$ is more complicated (although inspection of the same equations gives some clue), involving details of the numerical solution.

The lifetime $\tau_{\text {circ }}$ of the eccentric phase of the pre-LMXB is obviously also the lifetime of its orbital-modulation phase which we are investigating in this work. The sensitive dependence of this lifetime on the initial post-SN orbital parameters and the companion mass (through the scale parameter $\tau_{0}$ and due to the mass-dependence of $R_{\mathrm{c}}$ in Eq. (22)) makes for a wide range of possible values of this lifetime, $\sim 10^{3}-10^{8}$ years.

A crucial point is, of course, that if the companion is at or close to filling its Roche lobe at periastron in the post-SN orbit, it must remain so throughout most of this eccentric phase in order for the scenario to be self-consistent. The size of the Roche lobe at periastron is simply $p=a(1-e)$ multiplied by a well-known function of the mass ratio $q$. Since the latter does not change significantly during this phase, we need only study the evolution of the former. Our integration of the tidal-evolution equations show that, while $a$ and $e$ both decrease during this phase, $p=$ $a(1-e)$ decreases slowly through most of this phase, reaching a minimum and increasing thereafter at late stages. This is shown in Fig. 4 for the prototype 1E-like system displayed in Fig. 3 (see above). Thus, if the companion is initially at or close to filling its Roche lobe at periastron, it will remain so over most of this phase, and if it is inside its Roche lobe initially, it is likely to fill its Roche lobe later during this phase. It is also seen that Rochelobe contact ends at the last parts of this phase (when the orbit is nearly circular), since $p$ increases and becomes roughly constant there.

Thus, this tidal-evolution phase is a rough measure of the lifetime of Roche-lobe contact and orbital modulation of the propeller output. After this, the pre-LMXB becomes detached, and remains so until angular-momentum loss through gravitational radiation and/or magnetic braking brings it back to Rochelobe contact on a long timescale of $10^{8}-10^{9} \mathrm{yrs}$. We discuss this phase below in Sect. 6.4. 


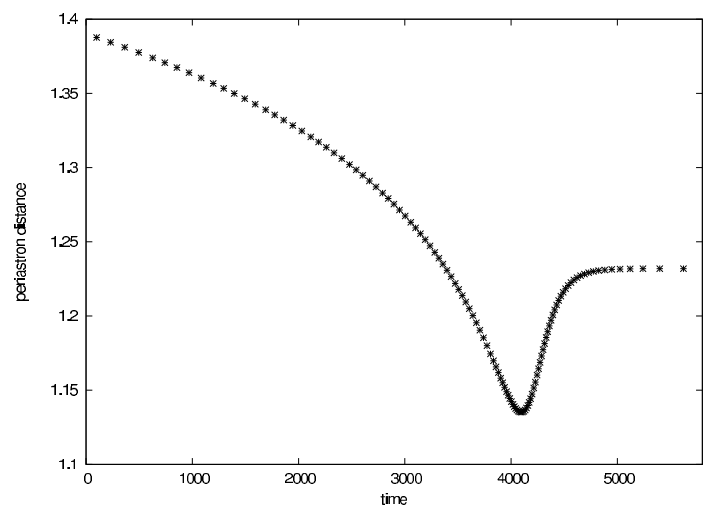

Fig. 4. Evolution of periastron distance $p=a(1-e)$ during tidal evolution of a prototype 1E-like system (see text). Shown is $p$ in units of the solar radius vs. time in years.

\subsection{Duration of propeller phase}

When the above tidal-evolution phase ends, is the neutron star still operating in the propeller phase? To answer this question, we consider the spindown of the neutron star from an initial spin period $P_{\text {spin }}^{i}$ to a final, longer spin period $P_{\text {spin }}^{f}$ under the action of the propeller torque given by either the IS-type torque (Eq. (1)) or the RUKL-type torque (Eq. (7)). In each case, this spindown is decsribed by

$\frac{\dot{\omega}}{\omega}=\frac{N}{I \omega}=\frac{1}{t_{\text {prop }}}$

where $I$ is the moment of inertia of the neutron star and $t_{\text {prop }}$ is the propeller spindown timescale.

First consider IS-type torques, for which $t_{\text {prop }}$ is given by

$t_{\text {prop }}=3 \sqrt{2} \frac{\left(2 G M_{x}\right)^{2 / 7} I}{\mu^{8 / 7} \dot{M}^{3 / 7}} \approx 2.5 \times 10^{5} \dot{M}_{14}^{-\frac{3}{7}} \mu_{30}^{-\frac{8}{7}} m_{x}^{\frac{2}{7}} I_{45} \quad \mathrm{yr}$,

where $I_{45}$ is $I$ in units of $10^{45} \mathrm{gm} \mathrm{cm}^{2}$, and other units are as before. Equation (24) can be integrated readily in this case, the total spindown time $\tau_{\text {prop }}$ from $P_{\text {spin }}^{i}$ to $P_{\text {spin }}^{f}$ being:

$\tau_{\text {prop }}=2.303 t_{\text {prop }} \log \left(P_{\text {spin }}^{f} / P_{\text {spin }}^{i}\right)$.

As discussed earlier, the ratio $P_{\text {spin }}^{f} / P_{\text {spin }}^{i}$ is believed to be in the range 10-100 (Ghosh 1995, and references therein), and its exact value does not matter because of the logarithmic dependence. On taking $m_{X}=1.4$ and the corresponding moment of inertia for a standard modern EOS, we arrive at

$\tau_{\text {prop }} \approx 2 \times 10^{6} \mathrm{yr}$

for canonical values of $\dot{M}, \mu$ and $I$.

Now consider RUKL-type torques, for which $t_{\text {prop }}$ is given by

$t_{\text {prop }}=t_{0}\left(P_{\text {spin }} / 0.01 \mathrm{~s}\right)$, where

$t_{0} \approx 2.3 \times 10^{7} \dot{M}_{14}^{-\frac{3}{8}} \mu_{30}^{-1.1} I_{45}$ yr.

Equation (24) can be integrated readily in this case also, the total spindown time $\tau_{\text {prop }}$ from $P_{\text {spin }}^{i}$ to $P_{\text {spin }}^{f}$ being:

$\tau_{\text {prop }}=t_{0}\left(P_{\text {spin }}^{f}-P_{\text {spin }}^{i}\right) /(0.01 \mathrm{~s}) \approx t_{0}\left(P_{\text {spin }}^{f} / 0.01 \mathrm{~s}\right)$, the second equality in the above equation coming from the fact that the ratio $P_{\text {spin }}^{f} / P_{\text {spin }}^{i}$ is believed to be in the range 10-100, as indicated above. The numerical value of $\tau_{\text {prop }}$ in this case is thus

$\tau_{\text {prop }} \approx 2.3 \times 10^{7} \dot{M}_{14}^{-\frac{3}{8}} \mu_{30}^{-1.1} I_{45}\left(P_{\text {spin }}^{f} / 0.01 \mathrm{~s}\right) \mathrm{yr}$,

which implies that, for canonical range $P_{\text {spin }}^{f} \sim 0.1-1 \mathrm{~s}$, as indicated in Sect. 2, we arrive at

$\tau_{\text {prop }} \approx 2 \times 10^{8}-2 \times 10^{9} \mathrm{yr}$

for canonical values of the variables $\dot{M}, \mu$ and $I$.

In comparing the total spindown times $\tau_{\text {prop }}$ given by the two types of torques, we notice that the time taken by the RUKLtype torque is $2-3$ orders of magnitude longer than that taken by the IS-type torque for identical values of $\dot{M}, \mu$ and $I$. This reflects the relative weakness of the RUKL-type torque discussed in Sect. 2. Next, comparing the values of $\tau_{\text {prop }}$ given by the above two types of propeller torques with the lifetime $\tau_{\text {circ }}$ of the eccentric phase given in the previous section, we reach the following conclusions. For the IS-type torque, we find that, over most of the parameter space, the neutron star would still be in the propeller phase at the end of the above tidal-evolution phase of the binary. For the RUKL-type torque, we find that this conclusion is valid over the entire parameter space. Thus, the RUKL-type torque makes the conclusion stronger.

As shown above, the companion has moved out of Rochelobe contact by the time that the tidal-evolution phase of the binary reaches conclusion, so that mass transfer stops, and so does the propeller action and its consequent soft X-ray production. Accordingly, throughout this first Roche-lobe contact phase, we expect the system to be in the propeller phase.

\subsection{Re-contact with Roche lobe and LMXB phase}

After orbit circularization and the loss of its first Roche-lobe contact, as described above, the pre-LMXB thus ceases to be an $\mathrm{X}$-ray source. But its orbit shrinks (i.e., the binary hardens) on a long timescale $\left(\sim 10^{8}-10^{9} \mathrm{yr}\right)$ due to two mechanisms of angular momentum loss from the system, viz., graviational radiation and magnetic braking (Ghosh 2007, and references therein). These are the standard mechanisms through which short-period pre-LMXBs are believed to harden, until Roche-lobe contact is regained and mass transfer restarts. But the transferred mass is now accreted by the neutron star, because its spin has been slowed down sufficiently over this long time that it acts as an accretor and not a propeller at the (large) mass-transfer rates that occur at this second Roche-lobe contact in the circularized binary. The system thus turns on as a canonical LMXB now, emitting strongly $\left(L \sim 10^{37}-10^{38} \mathrm{erg} \mathrm{s}^{-1}\right.$ ) in the canonical X-ray band characteristic of emission from the neutron-star surface, rather than the soft X-ray band characteristic of propeller emission from the vicinity of the magnetospheric boundary.

The timescale $t_{\mathrm{GR}}$ of orbit shrinkage due to gravitational radiation is given by (see, e.g., Faulkner 1971; Banerjee \& Ghosh 2006):

$t_{\mathrm{GR}} \approx 2 \times 10^{9} \frac{m_{\mathrm{T}}^{1 / 3}}{m_{x} m_{\mathrm{c}}}\left(\frac{P_{\mathrm{orb}}}{6.7}\right)^{8 / 3} \mathrm{yr}$

where $m_{\mathrm{T}} \equiv m_{x}+m_{\mathrm{c}}$, and all masses are in solar units. In this equation, we have scaled $P_{\text {orb }}$ to the value for $1 \mathrm{E}$, and substitution of the masses we have used above for this system gives $t_{\mathrm{GR}} \approx 4 \times 10^{9} \mathrm{yr}$. Generally, 1E-like systems with 
shorter periods and/or somewhat different companion masses will have $t_{\mathrm{GR}} \sim 10^{8}-10^{9} \mathrm{yr}$. Magnetic braking is believed to be comparable or weaker in strength to shrinkage by gravitational radiation at these orbital periods, so that the above estimate is a reasonable one for the 1E-type systems we have in mind here.

Thus, the system becomes a canonical, bright LMXB with a circular orbit and $P_{\text {orb }}$ in the range of, say, $2-10 \mathrm{~h}$. It is wellknown that systems with $P_{\text {orb }}$ exceeding about $12 \mathrm{~h}$ cannot come into Roche lobe contact by the above orbit-shrinkage mechanisms, since the time required would exceed the Hubble time, as Eq. (32) readily shows. However, these long-period systems also come into Roche-lobe contact eventually, as the low-mass companion completes its main-sequence evolution and expands. These systems thus also become canonical long-period LMXBs with circular orbits. The lifetime of this standard, bright LMXB phase is $t_{\mathrm{LMXB}} \sim 10^{8}-10^{9} \mathrm{yr}$.

\section{Discussion}

In this work, we have explored a pre-LMXB model of 1E, wherein the eccentric orbit of the very young pre-LMXB causes an orbital modulation in the mass-transfer rate, and the newborn, fast-rotating neutron star operates in the propeller regime, the propeller emission in soft X-rays following the above modulation after viscous smoothening in the accretion disk. In this section, we first discuss first some essential spectral and luminosity-dependent features of $1 \mathrm{E}$, and their connections with corresponding features in old, low-mass, soft X-ray transients (SXRTs) in their low/quiescent states, the prime example of this class being Aquila X-1 (Campana et al. 1998). Note that the well-known transient accretion-powered millisecond pulsar SAX J1808.4-3658 also shows a similar behavior (Stella et al. 2000). In these classes of low-mass X-ray binaries with old neutron stars, the neutron star is thought to operate in the propeller regime when the sources are in their low/quiescent states during decays of their outbursts. We then compare our model with the magnetar model which has been proposed recently for $1 \mathrm{E}$ (P08), and discuss how distinction between the two kinds of models might be attempted in future. Finally, we summarize our conclusions.

\subsection{X-ray spectra}

The XMM-Newton/EPIC (0.5-8 keV) X-ray spectra of $1 \mathrm{E}$ have been described by dL06. The time-averaged spectra from the 2005 low-state observations, when the source luminosity was $L \sim 10^{33} \mathrm{erg} \mathrm{s}^{-1}$, can be fitted by a two-component model consisting of a blackbody (BB) of temperature $k T_{\mathrm{bb}} \sim 0.5 \mathrm{keV}$ and an equivalent blackbody radius $R_{\mathrm{bb}} \sim 0.6 \mathrm{~km}$, plus a power-law (PL) of index $\Gamma \sim 3$, with $\sim 70 \%$ of the total flux coming from the blackbody component. Alternatively, the second component can also be a blackbody with a higher temperature. A re-analysis of the earlier 2001 XMM-Newton data, when $1 \mathrm{E}$ had a higher luminosity (by a factor $\sim 6$ ), yielded a similar two-component $(\mathrm{BB}+\mathrm{PL})$ model with essentially the same blackbody temperature $k T_{\mathrm{bb}}$ and power-law index $\Gamma$, but a larger equivalent blackbody radius $R_{\mathrm{bb}} \sim 1.3 \mathrm{~km}$, and a higher contribution from the PL component (the blackbody contribution was $\sim 50 \%$ of the total flux as opposed to the above $\sim 70 \%$ ), which made the overall spectrum harder (dL06).

We stress the remarkable similarity of the above observations with those of the spectra of SXRTs in their low/quiescent states (when the neutron stars in them are believed to be functioning in the propeller regime), taking the well-known source Aquila X-1 as the example. A detailed analysis of the BeppoSAX observations of Aquila X-1 in 1997 (Campana et al. 1998) has yielded the following results. At the lowest state, with source luminosity $L \sim 0.6 \times 10^{33} \mathrm{erg} \mathrm{s}^{-1}$, the (BB+PL) fit had a BB of temperature $k T_{\mathrm{bb}} \sim 0.3 \mathrm{keV}$ and an equivalent blackbody radius $R_{\mathrm{bb}} \sim 0.8 \mathrm{~km}$, plus a power-law (PL) of index $\Gamma \sim 1$, with $\sim 60 \%$ of the total flux coming from the blackbody component. As the luminosity increased by a factor $\sim 150$ to $L \sim 9 \times 10^{34} \mathrm{erg} \mathrm{s}^{-1}$, the $(\mathrm{BB}+\mathrm{PL})$ fit yielded a BB of temperature $k T_{\mathrm{bb}} \sim 0.4 \mathrm{keV}$ and an equivalent blackbody radius $R_{\mathrm{bb}} \sim 2.6 \mathrm{~km}$, plus a power-law (PL) of index $\Gamma \sim 1.9$, with $\sim 20 \%$ of the total flux coming from the blackbody component. Remembering that the total range of luminosities in these Aquila X-1 low-state observations during outburst decay is roughly $10^{33}-10^{35} \mathrm{erg} \mathrm{s}^{-1}$ (Campana et al. 1998), essentially identical to that of the 1E observations reported by dL06, the correspondence is very suggestive.

SXRTs are believed to be old systems with a neutron star and a low-mass companion in a close circular orbit, undergoing outbursts due to instabilties either in the accretion disk or in the mass supply from the low-mass companion. In their low/quiescent states during decays of outbursts, the fast-spining neutron star (spun up by accretion as per standard LMXB scenario) is believed to operate in the propeller regime. What we suggest in this work is that 1E-like systems are very young systems in the same regime: the young systems can show orbital modulation because of the orbital eccentricity, while the old systems are in circular orbit and cannot show such orbital modulation. However, the spectral signatures are very similar at similar luminosities, which supports our basic suggestion. We note that the timescales associated with $1 \mathrm{E}$ outburst appear to be $\sim 2-3$ years while those associated with Aquila X-1 outbursts appear to be $\sim 30-70$ days. It is possible that the basic phenomenon is rather similar in the two cases, and that the difference in detail is caused by the fact that accretion onto the neutron-star surface (with attendant high luminosities and hard X-ray spectra) does occur at the high states during the outbursts for old systems like Aquila X-1, but not for young systems like 1E.

A comprehensive theory of the emission spectra of propeller sources appears to be lacking, though Illarionov and co-authors have studied some effects of Comptonization in propellers in wind-accreting massive X-ray binaries (Illarionov \& Kompaneets 1990; Illarionov et al. 1993). Attempts at constructing such a theory for propellers in preLMXBs and in old LMXBs in low/quiescent states is clearly beyond the scope of this paper, and we shall confine ourselves here to the comment that the importance of Compton heating, considered in the above works on propellers in massive binaries, is also likely to be crucial for the systems we are focusing on in this work, as the observed power-law tails in the spectra at low luminosities suggest. These tails are particularly prominent in the low-state spectra of Aquila X-1 (Campana et al. 1998).

\subsection{Luminosity dependence of light curve}

dL06 have compared the 1E light curve in the 2005 low-state observations with that during the 2001 observations when the source luminosity was a factor $\sim 6$ higher. While the former light curve is relatively smooth with some cycle-to-cycle variations, the latter one shows more complex, somewhat "jagged" structure, with an occasional dip. Further, the pulsed fraction decreses from $\sim 43 \%$ to $\sim 12 \%$ as the luminosity increases. We discuss qualitatively how such features may arise. First, a propeller system is inherently more fluctuating than accreting system, because of a variety of fluctuations possible at the site of 
shock-heating and outflow. As mass-supply rate through the accretion disk increases, these fluctuations may increase, causing more complex profiles. Secondly, accretion disks in low-mass systems like LMXBs and CVs are thought to develop structures at their outer edges, which obscure emission from the compact object, and lead to dips. If these obscuring structures increase in size as mass-supply rate through the accretion disk increases, this would provide a natural explanation for the above appearance of the dips. Thirdly, as the mass-arrival rate $\dot{M}$ at $r_{\mathrm{m}}$ increases, $r_{\mathrm{m}}$ decreases (see Sect. 2), matter at the magnetospheric boundary becomes hotter, and the propeller becomes less supersonic, ultimately becoming subsonic. Now, it is well-known that the subsonic propeller torque $N_{\text {sub }} \sim \mu^{2} \Omega_{\mathrm{s}}^{2} / G M_{x}$ is independent of $\dot{M}$ (see Ghosh 1995, and references therein), and so will not follow the modulations of $\dot{M}$. Hence, as $\dot{M}$ and $L$ increase, the following phenomenon is likely to happen. As the upper limit of the excursions in $\dot{M}$ goes beyond the critical cross-over point from supersonic to subsonic propeller regime, the pulsed fraction will decrease because that part of $\dot{M}$ which is above this critical point will not contribute to the pulsed flux, and this decrease will increase with increasing $\dot{M}$. This may be a natural explanation for the above observation of reduced pulsed fraction at higher luminosity. More quantitative considerations will be given elsewhere.

\subsection{Comparison with magnetar model}

In a recent paper, P08 have described a model in which $1 \mathrm{E}$ is a magnetar, i.e., a neutron star with a superstrong magnetic field $\sim 10^{15} \mathrm{G}$ with a low-mass companion. The $6.7 \mathrm{~h}$ period is interpreted in this model as the spin period of the neutron star, the idea being that a neutron star with such strong magnetic field as above can be spun down to such long spin period, or such low spin frequency, in 2000 yrs. Magnetars are a fascinating possibility, and their relevance to soft gamma repeaters (SGRs) and possibly to anomalous X-ray pulsars (AXPs) has been the subject of much recent study. P08 have invoked an analogy with polars or AM Her-type cataclysmic variables containing white dwarfs with unusually strong magnetic fields, wherein torques acting on the magnetar spin it down in a short time to spin periods in close synchronism with the binary orbital period. In this analogy, they have been inspired by the similarity of the shape the $1 \mathrm{E}$ light curve to those of AM Her systems.

We have desribed in this work a model which does not require a neutron star with a superstrong magnetic field, but rather interprets the $6.7 \mathrm{~h}$ period as the orbital period of the binary system consisting of a neutron star with a canonical magnetic field of $\sim 10^{12} \mathrm{G}$ with a low-mass companion, the newborn, fast-rotating neutron star being in the propeller phase, and the propeller emission being modulated in the eccentric orbit of a young post-SN binary. We find that the observed $1 \mathrm{E}$ light curve can be quantitatively accounted for by our model. Our analogy is with propeller regimes of SXRTs like Aquila X-1 in their low/quiescent states, which we consider to be old, circularized analogues of $1 \mathrm{E}$ which are no longer orbitally modulated, but which have remarkably similar spectral properties. In this analogy, we have been inspired by the similarity between $1 \mathrm{E}$ and the SXRTs in both the spectral characteristics and their changes with source luminosity, as well as the shapes of the outbursts and the way in which propeller-like properties emerge at low luminosities during outburst decays.

An interesting question is that of possible discriminators between the above two models. It appears to us that if all observed properties of $1 \mathrm{E}$ and similar systems can be accounted for by known characteristics of early stages of pre-LMXBs born according to the standard CE evolution and He-star supernova scenario, such as we have described in this paper (or by other possible models involving standard evolutionary scenarios), there would not be any compelling need for invoking exotic objects like magnetars for this class of objects. On the other hand, if one finds unique observed features in this class of objects that cannot be explained at all within the framework of standard evolutionary scenarios, presence of magnetars in such objects may well be hinted at. However, answering this question is beyond the scope of this paper: we are pursuing the matter, and the results will be reported elsewhere.

\subsection{Conclusions}

The work reported here suggests that 1E-type systems are early stages of pre-LMXBs born in the SN of He-stars in binaries of (He-star + low-mass star) produced by common-envelope (CE) evolution. As long as the post-SN binary is eccentric, and the neutron star is in the propeller regime, soft X-ray emission modulated at the orbital period may be expected to occur. As the orbit circularizes, modulation would stop, and as the low-mass companion moves out of Roche-lobe contact, the source would not be observed in X-rays. The companion would come into Rochelobe contact again on a long timescale due to orbit shrinkage by emission of gravitational radiation and magnetic braking, and/or by the evolutionary expansion of the companion. This would lead to a standard LMXB: an old neutron star in circular orbit with a low-mass companion. Thus, steady-state arguments, with lifetimes of 1E-type systems estimated at $\sim 10^{6}-10^{7} \mathrm{yrs}$ and those of LMXBs estimated at $\sim 10^{8}-10^{9}$ yrs, would lead us to expect $\sim 1$ 1E-type systems per $\sim 100$ LMXBs, which is consistent with current observations. However, we must be careful here, as these are overall arguments for the whole population. If one specifically investigates young supernova remnants (SNRs), the chances of finding such systems may be considerably higher, since eccentric binary systems are to be found preferentially in such SNRs. More detailed considerations will be given elsewhere.

The lifetime of the eccentric-binary phase may be increased by an effect we have not included in this introductory work. The effect is that of an enhancement of eccentricity when mass and angular momentum are lost from a binary system which is already eccentric. This dynamical effect is well-known in the literature (see, e.g., Huang 1963) and its applications to compact X-ray binaries have been made earlier (Ghosh et al. 1981). For an eccentric compact binary with the neutron star in the propeller regime leading to the loss of both mass and angular momentum from the system, such considerations are applicable. However, it is possible that, at the rates of mass transfer and loss inferred for 1E-type systems, this effect is a minor one.

Several lines of further investigation are naturally suggested by the considerations we have given in this paper. Foremost among them is a theory of the spectral characteristics of propeller emission in disk-fed propeller systems. This would help clarify the remarkable spectral similarity (including changes in spectral parameters with luminosity) between 1E and SXRTs like Aquila X-1 in their low/quiescent state, as described in Sect. 7.1. A search for point soft X-ray sources in other young SNRs would clarify the observational situation greatly. We note that these sources may or may not be periodically modulated, as we have argued in Sect. 7.2 that such modulations may decrease and disappear in certain luminosity states. However, the spectral characteristics would still be a most valuable diagnostic. 
These and other investigations are under way, and results will be reported elsewhere.

Acknowledgements. It is a pleasure to thank A. de Luca for sending data on the light curves, to thank E. P. J. van den Heuvel and L. Stella for stimulating discussions, and to thank the referee for comments which improved the paper considerably.

\section{References}

Avni, Y. 1976, ApJ, 209, 574

Bath, G., \& Pringle, J. 1981, MNRAS, 194, 967

Brown, J., \& Boyle, C. 1984, A\&A, 141, 369

Campana, S., Stella, L., Mereghetti, S., et al.1998, ApJ, 499, L65

Davies, R., \& Pringle, J. E. 1981, MNRAS, 196, 209

Davies, R., Fabian, A. C., \& Pringle, J. E. 1979, MNRAS, 186, 779

de Luca, A., Caraveo, P. A., Mereghetti, S., Tiengo, A., \& Bignami, G. F. 2006, Science, 313, 814 (dL06)

de Luca, A., Mignani, R. P., Zaggia, S., et al.2008, ApJ, 682, 1185

Faulkner, J. 1971, ApJ, 170, L99

Ghosh, P. 1995, ApJ, 453, 411 (G95)

Ghosh, P. 2007, Rotation and Accretion Powered Pulsars (Singapore: World Scientific Publications)

Ghosh, P., Elsner, R. F., Weisskopf, M. C., \& Sutherland, P. G. 1981, ApJ, 251, 230

Heyl, J., \& Hernquist, H. 2002, ApJ, 567, 510

Huang, S.-S. 1963, ApJ, 138, 471

Illarionov, A. F., \& Kompaneets, D. A. 1990, MNRAS, 247, 219

Illarionov, A. F., \& Sunyaev, R. A. 1975, A\&A, 39, 185 (IS75)

Illarionov, A. F., Igumenschev, I. V., \& Kompaneets, D. A. 1993, The evolution of X-ray Binaries, ed. S. S. Holt, \& C. S. Day (New York: AIP), 601
Joss, P., \& Rappaport, S. 1984, ARA\&A, 22, 537

Kalogera, V. 1996, ApJ, 471, 352

Landin, N. R., Mendez, L. T. S., \& Vaz, L. P. R. 2009, A\&A, 494, 209

Lightman, A. 1974, ApJ, 194, 429

Lovelace, R. V. E., Romanova, M. M., \& Bisnovatyi-Kogan, G. S. 1999, ApJ, 514, 368 (LRB99)

Lubow, S., \& Shu, F. 1975, ApJ, 198, 383

Lynden-Bell, D., \& Pringle, F. 1974, MNRAS, 168, 603

Meibom, S., \& Mathieu, R. D. 2005, ApJ, 620, 970

Mignani, R., et al. 2007 [arXiv:0711.0113]

Mineshige, S., Rees, M. J., \& Fabian, A. C. 1991, MNRAS, 251, 555

Pavlov, G., et al. 2002, Neutron Stars in Supernova Remnants, ASP Conf. Ser., 271,2002

Pizzolato, F., Colpi, M., De Luca, A., Mereghetti, S., \& Tiengo, A. 2008, ApJ, 681, 530 (P08)

Pravdo, S., \& Ghosh, P. 2001, ApJ, 554, 383

Pringle, J. 1981, ARA\&A, 19, 137

Romanova, M. M., Ustyugova, G. V., Koldoba, A. V., \& Lovelace, R. V. E. 2004, ApJ, 616, L151

Romanova, M. M., Ustyugova, G. V., Koldoba, A. V., \& Lovelace, R. V. E. 2005, ApJ, 635, L165 (RUKL05)

Shakura, N., \& Sunyaev, R. 1973, A\&A, 24, 337

Stella, L., Campana, S., Mereghetti, S., Ricci, D., \& Israel, G. L. 2000, ApJ, 537, L115

Tuohy, I., \& Garmire, G. 1980, ApJ, 239, L107

Ustyugova, G. V., Koldoba, A. V., Romanova, M. M., \& Lovelace, R. V. E. 2006, ApJ, 646, 304 (UKRL06)

Verbunt, F., \& Zwaan, C. 1981, A\&A, 100, L7

Zahn, J.-P. 1977, A\&A, 57, 383

Zahn, J.-P. 1978, A\&A, 67, 162 